\title{
Systematic Development of Control Designs via Formal Refinement
}

\author{
Manoranjan Satpathy ${ }^{1}$, Colin Snook ${ }^{2}$, Silky Arora ${ }^{1}$, S. Ramesh ${ }^{1}$ and Michael Butler ${ }^{2}$ \\ ${ }^{1}$ General Motors Global $R \& D$, International Tech Park (ITPL), Bangalore - 560066, India \\ ${ }^{2}$ School of Electronics \& Computer Science, University of Southampton, Highfield SO17 1BJ, UK
}

\begin{abstract}
Keywords: $\quad$ Refinement based development: Discrete Controllers: Simulink/Stateflow, Event-B
Abstract: The Simulink/Stateflow (SL/SF) modeling framework is widely used in industry for the development of control applications. However, such models are not amenable to formal reasoning. Controllers can also be designed using formal specification languages. Such designs can be formally verified, but the models do not explicitly represent control or data flow information. In this paper, we discuss RRM diagrams (RRMDs), a new modelling notation which incorporates the benefits of these two formalisms. RRMDs are graphical formal models and they also support incremental formal development. We have used synchronising state machines to encode RRMDs. We have also developed a prototype tool which translates RRMDs automatically to SL/SF designs.
\end{abstract}

\section{INTRODUCTION}

The SL/SF modeling framework is widely used in industry for the development of control applications. Its advantages are: (a) a model being graphical is better understood to engineers, and (b) a model being executable eliminates many design level bugs. The disadvantages are: (a) the modelling language does not have a formal semantics and hence models are not amenable to formal reasoning, (b) models are directly created from requirements, this is a big step and errors are likely to creep in, and (c) validation can only be done a posteriori.

Controllers can alternatively be built using formal specification languages like Event-B (Abrial, 2010). The advantages are that (a) formal languages support incremental design, and (b) any design step can be verified as and when it is introduced. The disadvantages are that (a) the languages are text-based, and so the intuition behind a model is not explicit, and (c) the models do not explicitly represent control or data flow information which is counter-intuitive to a modelling engineer.

In this paper, we discuss RRM diagrams (RRMDs) which takes the advantages of both the above formalisms. Models as RRMDs are graphical; this formalism supports incremental feature augmentation and design. Figure 1 outlines the overall development process using RRMDs. We consider a fragment of certain requirements and build a RRMD, say R1. This RRMD has an equivalent representation in Event-B, say M1. M1 is checked for consistency using tool support. Next some more require-

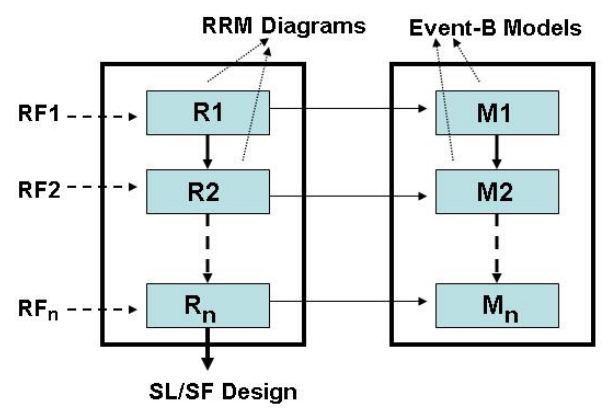

Figure 1: The Overall Development Process

ments are selected and R1 is extended (or refined) to obtain RRMD R2. Let M2 be the Event-B representation of R2; the refinement relationship between M1 and M2 is verified using tool support. Like this RRMDs - and so their Event-B models - are refined until all requirements are incorporated. Ambiguities and missing requirements are discovered in the process. From the final RRMD, a SL/SF design can be auto-generated, which we can claim to be correct-byconstruction. The main contributions of our paper are:

- Formulation of RRMDs as a graphical formal modelling notation for developing control designs.

- Use of the UML-B tool (Snook and Butler, 2006; Snook and Butler, 2008) for encoding and animating RRMDs. The tool makes it possible to derive Event-B models automatically from the RRM diagrams.

- Implementation of a translator which automati- 


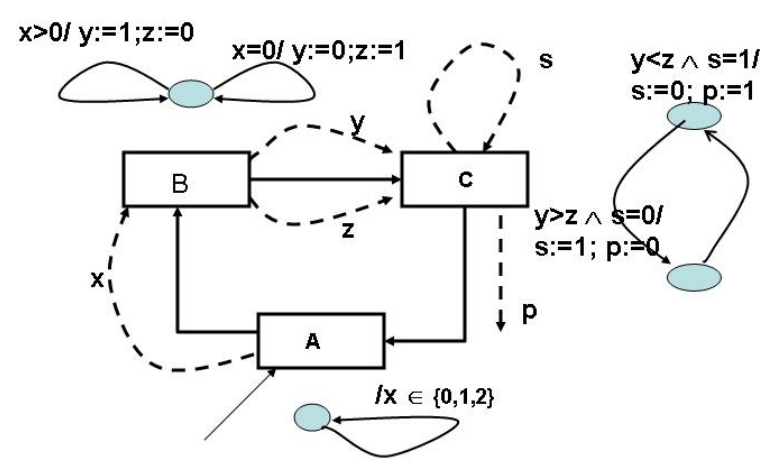

Figure 2: An RRM Diagram; $\mathrm{C}$ is a state holding block.

cally derives SL/SF designs from RRM diagrams.

\section{EVENT-B AND UML-B}

The Event-B method is a formal development method for modelling and development of distributed systems. An Event-B model consists of state variables and invariants. In addition, there are events, each has a guard and an action. The state variables are first initialised. Thereafter, one of the enabled events is selected non-deterministically for execution. This continues as long as there are enabled events. That the events preserve the model invariants can be checked using tool support. An abstract model can be refined; the refinement relationship between the abstract and the concrete models can be checked using tool support. Further details can be found in (Abrial, 2010). A RRMD has an equivalent representation in Event$\mathrm{B}$; hence RRMDs get the benefit of the sound theory and tool support of Event-B.

UML-B is a visual 'front-end' for the Event-B notation and includes a state machine diagram editor. Tool support for UML-B is provided by a plugin to the Rodin platform (Abrial et al., 2006). State machines may be refined by adding nested state machines and can be animated via a plug-in that utilises the Pro-B (Leuschel and Butler, 2003) model checker and animator. We will encode our RRMDs as UML-B models.

\section{RRM DIAGRAMS}

Figure 2 shows an RRM diagram, which consists of blocks and connectors. Connectors are of two kinds: control flow and data flow edges. In the figure, they have been respectively shown as solid and dashed

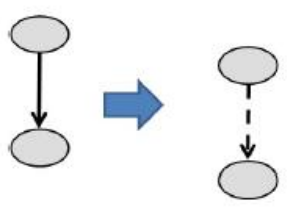

(a)

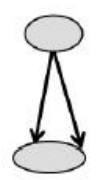

(b)

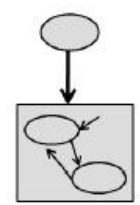

(c)

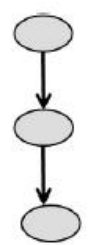

(d)
Figure 3: Refinement of RRMD blocks

lines. A control flow edge determines a block execution order, and a labelled data flow means the source block computes the value of the labelled variable and the target block uses it. Within a block, some computation is performed which is represented as a state machine; each transition has a guard and an action. Before a RRMD is made to execute, all variables in it are initialized. There are 3 computations blocks identified by $\mathrm{A}, \mathrm{B}$ and $\mathrm{C}$ in Figure 2. A is the start block from which computation starts. The control flow edges signify that B is computed after A, C is after B, and A is after $\mathrm{C}$ and so on.

The state machines for the respective blocks are as shown in the figure. When a block starts execution, one of the enabled transitions is executed. Within a state machine, a sequence of transitions executes till there is no enabled transition. In such a situation, control moves to the next block. In the figure, the lone transition in block $\mathrm{A}$ assigns a non-deterministic value (from the set $\{0,1,2\}$ ) to variable $x$; next, the state machine finishes execution and control moves to block $\mathrm{B}$. There are additional flags which disable the state machine of $\mathrm{A}$ and enables the transition(s) in block $\mathrm{B}$; in the figure, they have not been shown to avoid clutter. In block $\mathrm{B}$, either of the transitions is executed which uses variable $x$ and produces output variables $y$ and $z$. Next block B finishes execution flag variables ensure this - and control moves to block C. Block $\mathrm{C}$ uses $y$ and $z$ and produces output variables $p$ and $s$. Variable $s$ is produced as well as consumed (in the next cycle) by block $\mathrm{C}$, in this sense this is a state-holding block, the dashed self-loop signifies that. $p$ is an open output, not yet used by any other block, possibly, a latter refinement would use it.

\subsection{Refinement of an RRMD}

Refinement of an RRMD involves (a) refinement of block state machines, (b) closing of open inputs and outputs, and (c) sequencing of parallel control.

Refinement of State Machines: A refinement of a block state machine can be any of (a) strengthening of a transition action, (b) parallel splitting of a transi- 


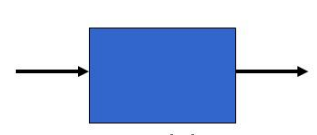

(a)

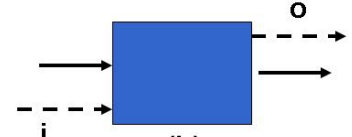

(b) (c)

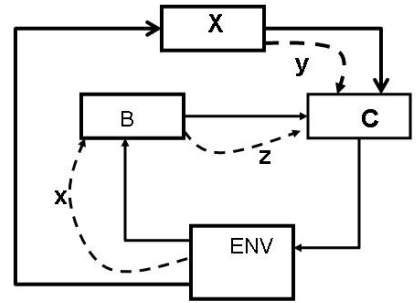

Figure 4: Closing of open inputs and outputs

tion (c) sequential splitting of a transition, and (c) creation of sub-states and lifting of the transitions at the parent level to the sub-state level. In Figure 3, (a) represents strengthening of a transition action, (b) shows the case splitting of a single transition into a number of parallel transitions, (c) shows creation of sub-states and transitions within a state, and (e) shows sequential splitting of a transition; the first transition produces a value and the second one uses it. We generate EventB models from RRMDs. What refinement steps we perform at the RRM level, it is checked that they are meaningful in the context of Event-B.

Handling of open inputs and outputs: State machine refinement of a RRMD block may result in creation of new state variables; they will be represented as open inputs and outputs; see Figure 4(b). An open input can be any of (i) an environmental input, (ii) the input is already produced by another block, or (iii) this input is not created anywhere. Our aim is to build control applications, so, we assume in an RRMD, there is a special block representing the environment, say ENV. If the open input is from the environment then this is closed by making it an output of ENV. If the open input is an output of another block, then this input is closed by connecting it by a data flow edge to this block. If the open input is not from the environment and it is not yet produced by any of the blocks, this has to be an internal input, and it would be computed in the subsequent modelling steps. So we create a new block which generates this open input. Refer to Figure 4(c) in which the open input $y$ has been closed by creating a new block X parallel to the existing control, block X gives a non-deterministic value to $y$. The parallel control path is created between ENV and the block which requires this input. Note that the nondeterminism in block X will be refined at a later stage. Sequencing of Parallel Control: Two parallel control paths would mean the two paths can be executed in arbitrary order. So sequencing them can be a refine-
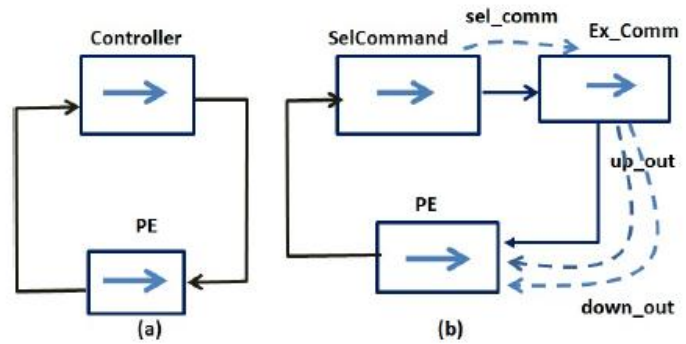

Figure 5: (a) The initial RRMD, (b) The first refined RRMD

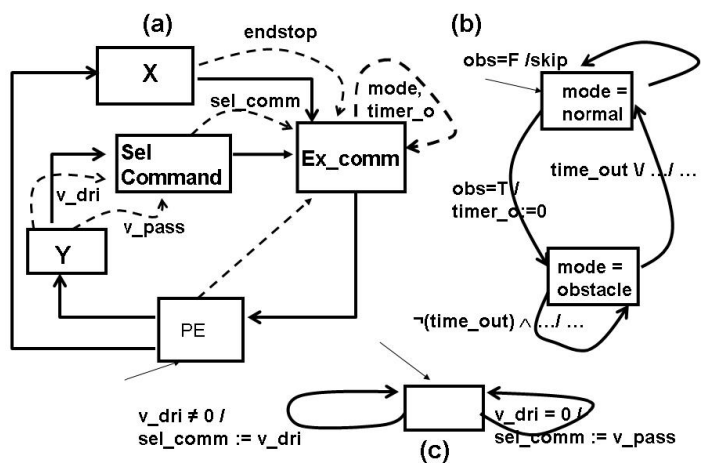

Figure 6: (a) RRMD after obstacle modeling, (b) state diagram of Ex_comm, (c) state diagram of SelCommand

ment step. In Figure 4(c), blocks B and X are in two parallel control paths; they can be made sequential depending on the data dependency between them.

\section{DESIGN USING RRMDS}

For developing control applications, we make certain assumptions: (a) the plant, user commands, sensors and actuators are abstracted by a special RRM block called PE (for plant \& environment), and (b) there is a sampling interval in which inputs arrive, processing is done by the controller, and output goes to the actuator. Time constraints are modelled with respect to this sampling interval. We consider the RRMD of Figure 5(a) as the initial abstract model of any control design. Block Controller abstracts the controller, and $\mathrm{PE}$ as we have discussed earlier. There are no data flow edges; the control flow edges model that the execution control alternates between PE and Controller.

\subsection{Case Study}

A Power Window Controller with obstacle detection capability (PWC-OD) will be our case study. Its requirements (Mathworks, 2012) are outlined as follows: R1) Both driver and passenger can control glass 
door movements using their own up/down switches, R2) When the glass is at the top (bottom) position then the up (down) command will not have any impact, R3) A driver command has higher priority over a passenger command; when both up and down switches are pressed (by driver or passenger) at the same time, it will be considered as if no switch has been pressed, R4) When the window is moving up, and an obstacle is detected, the glass moves down for a certain duration or when the lower end of the window is reached; During this time, commands from the driver or the passenger are ignored, and R5) If an up button is pressed and released before a threshold time limit, then it is interpreted as an auto-up command, and the window rolls up to its top limit; however, if the button is pressed for more than the threshold value, then the glass moves up step by step till the button is released or the top limit is reached; similar behaviour occurs when the down button is pressed.

\subsection{Incremental Modeling}

We will refine the Controller block in Figure 5(a); Figure 5(b) shows the refined RRMD after the following elementary refinements: (a) block Ex_Comm (Execute Command) is a refinement of Controller which takes the open input sel_comm (selected command), and produces the outputs up_out (glass to go up) and down_out (glass to go down) to be fed to the actuator, and (b) the open input sel_comm is closed by making it an output of SelCommand (Select Command).

In the second refinement, we make the block SelCommand deterministic. This relates to the requirement that driver commands have higher priority. This block receives two open inputs v_dri and v_pass (for validated driver and passenger commands). When $\mathrm{v} \_d r i$ is present, sel_comm gets the value of $\mathrm{v}$ _dri; otherwise, it gets the value of $v_{-}$pass. The state machine in Figure 6(c) models this behaviour.

Next we refine Ex_comm based on the requirement of the obstacle conditions. The state diagram of the Ex_comm block models this behaviour; see Figure 6(b). A new variable mode models that control can be either in normal or obstacle mode. When in normal mode, and an obstacle is detected, the mode changes to obstacle mode. Control stays there till time out occurs or the bottom window limit is reached. Thereafter, control moves to normal mode. Figure 6(a) shows the new RRMD. The state diagram of Ex_comm has four transitions and two new state variables: timer_o and mode; timer_o models the progress of time. The loop around Ex_comm shows these variables (Figure 6(a)); Ex_comm is thus a state holding block. The transitions require two extra inputs obs - whether obstacle is present - and endstop - whether any of the top limits is reached. The former being an environmental input originates from $\mathrm{PE}$, and the latter is an internal input. Therefore, we create a new block ( $\mathrm{X}$ in the figure) in the control flow path between PE and Ex_comm to produce the value of endstop.

The block Ex_comm is further refined to model the behaviour as specified by requirement $R 5$, the details are beyond the scope of this paper.

\section{TOOL SUPPORT FOR RRMDS}

The controller executes in two orthogonal dimensions. Firstly, the controller executes in a cyclic loop, acquiring inputs, processing them and then making some decisions about control. Simultaneously and independently the controller can be thought of as progressing in a control mode dimension. In this dimension the control responds to inputs by changing its state and consequent behaviour. The control mode behaviour progresses every time the processing loop is ready to make decisions about control. We model a RRMD by synchronising state machines in UML-B, this we illustrate through an example.

Figure 7 is the UML-B representation of the RRMD in Figure 6. In the UML-B syntax, control flow edges become states, and blocks of the RRMD become transitions. For example, block PE has become a single transition (state machine of PE has a single transition). The block SelCommand has two transitions, so two parallel transitions in the UML-B model: selectDri and selectPass. This kind of encoding is true when blocks are not state-holding. When a block is state-holding as in case of Ex_comm, we create an orthogonal state machine (Figure 7). The state machine in the left encodes the outer control loop behaviour of the RRMD in Figure 6(a); the state machine in the right is same as the block state machine of Ex_comm. In the first state machine of the UML-B model in Figure 7, the single transition between states PRIORITIZED and ENVIRONMENT represents the

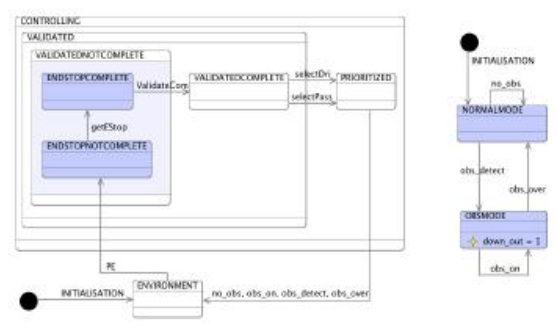

Figure 7: UML-B representation of the RRMD in Figure 6 
block Ex_comm, and this single transition is labelled with all the transition names of the state machine in the mode state machine (in the right). During execution, both these state machines are synchronized on the same transition labels.

Each RRMD corresponds to an Event-B model. The UML-B tool can make this translation. In a UML-B model, invariants can be added to the states of the UML-B state machine. Upon translation, these invariants are directly lifted to the Event-B model. Tools like Rodin Platform (Abrial et al., 2006) can be used to discharge the proof obligations. The UML-B model and Event-B translation with proofs are available as a Rodin archive (Snook, 2012).

\section{RRMD TO SL/SF}

In a fully developed RRMD, all blocks excepting the $P E$ are deterministic; PE gives non-deterministic values to environmental outputs. We next remove the control flow edges because at this stage, data flow edges determine the control flow. Next if we remove block PE and ignore the block internals, then it looks like a SL/SF model; i.e., if we view it as a SL/SF model, the inter-block connections would remain the same. In other words, if we translate the block internals to Simulink subsystems, then we would get an equivalent SL/SF model.

\subsection{RRM Block to Simulink Subsystem}

If a RRMD block is state-holding, then we make it a Stateflow subsystem. The structure of Stateflow chart remains exactly the same as the block state machine. The guard/action of a transition in the block state machine becomes the guard/action of the corresponding transition in the Stateflow chart; however, the Stateflow syntax needs to replace the RRMD syntax. If a block is not state-holding, we translate this block state machine to a Simulink subsystem. Based on the

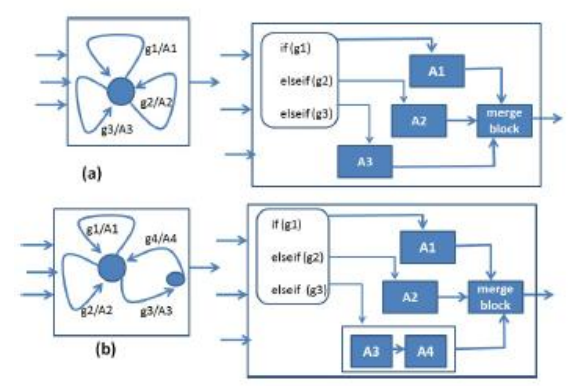

Figure 8: RRMD blocks to Simulink subsystems-I pattern of the state machine, we define the following mapping functions:

- If the pattern is as in Figure 8(a), then based on the guards of the transitions, an if-else-if Simulink block is created. In the figure, guards g1, g2 and $\mathrm{g} 3$ are mutually exclusive (this is by design and this property is proved within Event-B), and they become the conditions in the if-elseif-else Simulink block; its outputs trigger the subsystems for the transition actions, and the outputs of such subsystems are fed to a merge Simulink block; refer to the figure. The guards and actions are translated to the Simulink syntax.

- If the pattern is as in Figure 8(b), the translation is similar excepting the sequencing of the subsystems corresponding to actions A3 and A4.

- If the pattern is of type as in Figure 9, then the translation leads to a hierarchy of if-else-if blocks as shown in the same figure.

\subsection{SL/SF Translation Tool}

The UML-B to SL/SF translator is available as a toolbar button that is enabled when a UML-B statemachine is selected in the Rodin editor view. The state-machine must represent an RRMD top-level diagram. The translator tool generates a MATLAB m-script text file containing commands that build a SL/SF model. The m-script file can be loaded using the MATLAB command window. Figure 10 shows the auto-generated SL/SF model for the PWC-OD, and Figure 11 shows the Stateflow chart of the subsystem ExecuteCommand (EX_comm in RRMD).

\section{ANALYSIS OF OUR METHOD}

SL/SF design generation: At present, most of the controllers are developed using SL/SF designs. Engineers are very comfortable with SL/SF; many validation frameworks like model-in-loop, processor-inloop etc. exist around SL/SF designs. If engineers are

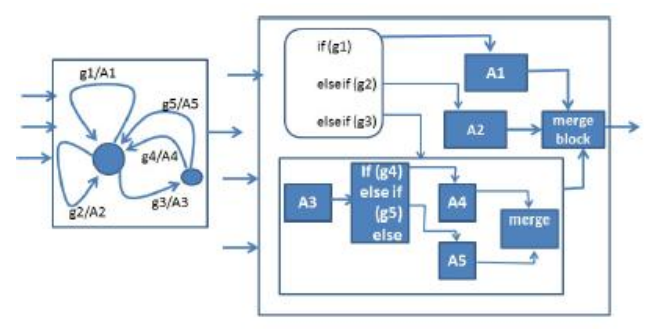

Figure 9: RRMD blocks to Simulink subsystems-II 


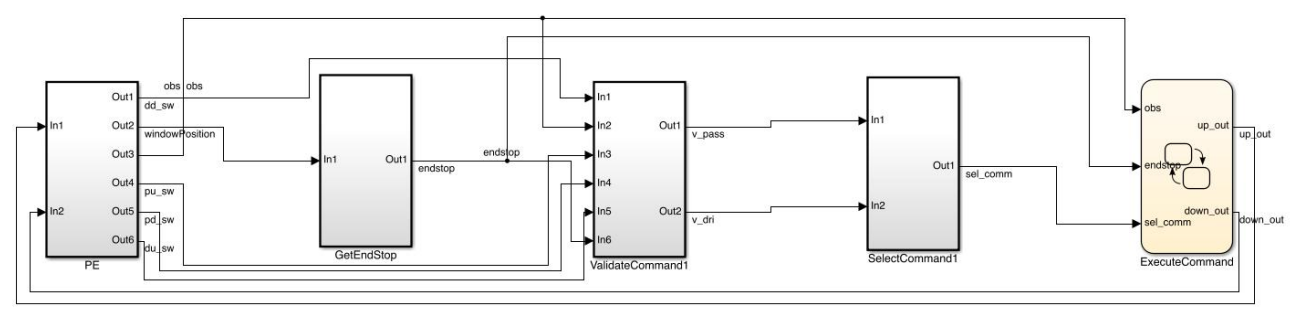

Figure 10: SL/SF design of PWC-OD, auto-generated from its RRMD

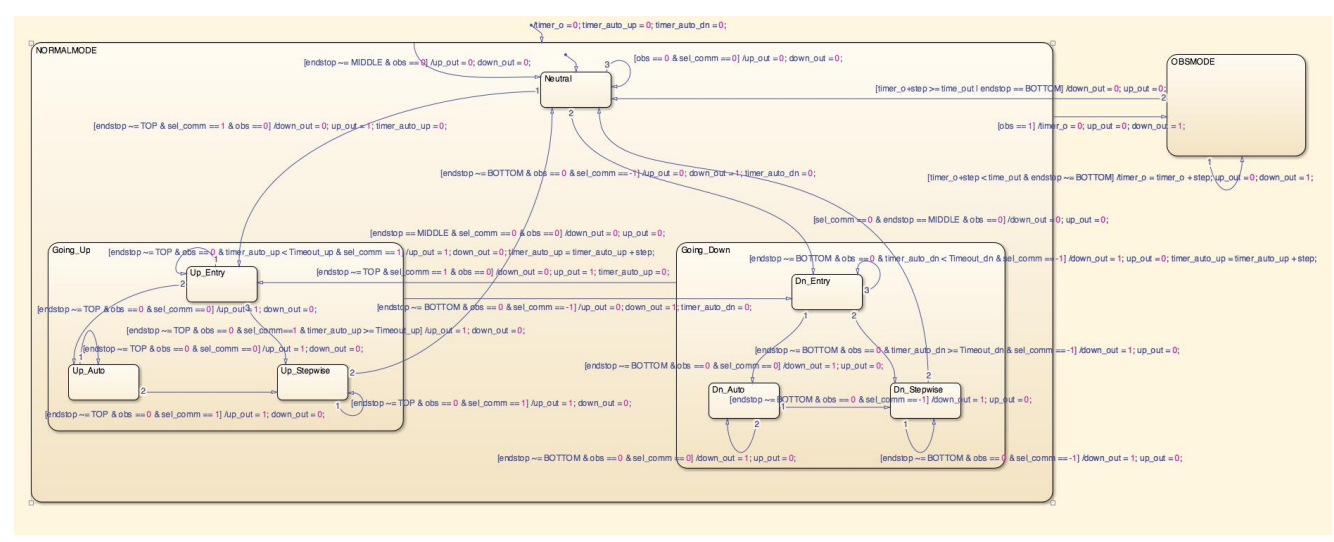

Figure 11: Stateflow chart of the ExecuteCommand subsystem in Figure 10

supplied with high quality SL/SF designs, they would readily accept those; the same cannot be said if code is directly given to them. Our work provides a way of introducing formal methods in current industrial practice, thus it contributes to the formal methods community. We also contribute to the SL/SF community; at present models are obtained manually, whereas our method generates them automatically.

Problems with Manual Generation: Generating SL/SF designs directly from requirements is a big intellectual step, and the process can be error-prone. Since we use formal refinements, and invariants are proved using tool support, we can claim that the SL/SF designs that we generate are of high quality.

Refinements of RRMDs: We have observed from five industrial case studies that refinements defined over RRMDs lead to valid refinements in Event-B. Formalisation of RRMD refinements is a part of our future work.

\section{CONCLUSIONS}

In this paper, we have shown how Event-B models can be generated in a systematic manner using RRMDs. RRMDs offer a visual perspective to the whole development process. Through an automotive case study, we have shown how RRMDs can deal with requirements in a systematic manner. Our approach also provides a systematic way of obtaining SL/SF control designs from requirements.

\section{REFERENCES}

Abrial, J.-R. (2010). Modeling in Event-B - System and Software Engineering. Cambridge University Press.

Abrial, J.-R., Butler, M. J., Hallerstede, S., and Voisin, L. (2006). An open extensible tool environment for event-b. In ICFEM, pages 588-605.

Leuschel, M. and Butler, M. (2003). Prob: A model checker for b. In FME 2003: FORMAL METHODS, LNCS 2805 , pages $855-874$. Springer.

Mathworks (2012). Simulink power window controller specification, http://www.mathworks.co.uk/help/simulink/examples.

Snook, C. (2012). Power window case study models in uml-b and event-b with generated simulink model, http://eprints.soton.ac.uk/345699/.

Snook, C. and Butler, M. (2006). Uml-b: Formal modeling and design aided by uml. ACM Transactions on Software Engineering and Methodology (TOSEM), 15(1):92-122.

Snook, C. and Butler, M. (2008). Uml-b and event-b: An integration of languages and tools. In IASTED International Conference on Software Engineering. 